\title{
A New Three Dimensional Dissipative Boussinesq Equation for Rossby Waves and Its Multiple Soliton Solutions $\rrbracket$
}

\section{Liguo Chen}

Inner Mongolia University of Finance and Economics

Liangui Yang ( $\nabla$ lgyang@imu.edu.cn )

Inner Mongolia University https://orcid.org/0000-0002-6218-1316

\section{Research Article}

Keywords: Rossby waves, Boussinesq equation, reduced perturbation method, trial function method, auxiliary equation method

Posted Date: March 4th, 2021

DOI: https://doi.org/10.21203/rs.3.rs-262870/v1

License: (c) (i) This work is licensed under a Creative Commons Attribution 4.0 International License. Read Full License

Version of Record: A version of this preprint was published at Results in Physics on July 1st, 2021. See the published version at https://doi.org/10.1016/j.rinp.2021.104389. 


\title{
A new three dimensional dissipative Boussinesq equation for Rossby waves and its multiple soliton solutions ${ }^{\text {th }}$
}

\author{
Liguo Chen ${ }^{\mathrm{a}}$, Liangui Yang ${ }^{\mathrm{b}, *}$ \\ ${ }^{a}$ School of Statistics and Mathematics, Inner Mongolia University of Finance and \\ Economics, Hohhot, 010070, China \\ ${ }^{b}$ School of Mathematical Sciences, Inner Mongolia University, Hohhot, 010021, China
}

\begin{abstract}
A new three dimensional nonlinear dynamic theoretical model is derived from fluid mechanics system. In this paper, From the quasi-geostrophic barotropic potential vorticity equation, we obtain a three dimensional dissipative Boussinesq equation by the reduced perturbation method, i.e. $u_{t t}+e_{1} u_{x x}+e_{2}\left(u^{2}\right)_{x x}+$ $e_{3} u_{t x y}+e_{4} u_{x x x x}+e_{5} u_{x x y y}=0$. It is emphasized that the new equation is different from the existing Boussinesq equations, which describe the three dimensional nonlinear Rossby waves in the atmosphere. Moreover, we explore the dispersion relation of the linear wave through the new equation. Using the trial function and auxiliary equation method, the two kinds of soliton solutions of the equation are obtained successfully. Finally, the formation mechanism of Rossby waves is discussed by multiple soliton solutions.

Keywords: Rossby waves, Boussinesq equation, reduced perturbation method, trial function method, auxiliary equation method
\end{abstract}

\section{Introduction}

In the 20th century, Carl-Gustaf Rossby, a famous meteorologist, discovered a slow, large-scale wave in the Earth's atmosphere, with a wave length of

\footnotetext{
* Corresponding author

Email address: lgyang@imu.edu.cn (Liangui Yang)
}

Preprint submitted to Journal of ${ }^{A} T_{E} X$ Templates

February 26, 2021 
$3000 \mathrm{~km}$ to $10000 \mathrm{~km}$, and named Rossby waves. It is a natural phenomenon in the Earth's atmosphere, which is caused by the earth's rotation. Many studies have shown that the evolution of Rossby waves is closely related to extreme weather phenomena, such as the Walker circulation, ${ }^{[1]}$ atmospheric blocking, ${ }^{[2]}$ the low-frequency Madden-Julian oscillation, ${ }^{[3]}$ the ENSO. ${ }^{[4,5]}$ Therefore, Rossby waves have been paid more and more attention from researchers. The formation and evolution of Rossby waves become an important theoretical basis for the weather forecast. In the last decades, researchers used to study the physical factors affecting Rossby waves by building various nonlinear partial differential equation, such as the Korteweg-de $\operatorname{Vries}(\mathrm{KdV})$ equation, modified Korteweg-de Vries(mKdV) equation, ${ }^{[6-9]}$ Benjamin-Ono(BO) equation, ${ }^{[10]}$ Benjamin-DavisOno(BDO)equation, ${ }^{[11]}$ BDO-Burger equation, ${ }^{112]}$ Schrödinger equation, ${ }^{[13,14]}$ Boussinesq equation, ${ }^{[15,16]}$ Boussinesq-BO equation ${ }^{[17]}$ and so on, which describe $(1+1)$-dimensional nonlinear Rossby waves.

In recent years, a variety of three dimensional equations are obtained to characterize the formation of Rossby waves, in order to explain some weather phenomena more accurately in real atmosphere motions. In barotropic atmosphere, from the quasi-geostrophic potential vorticity equation(QGPVE) describing Rossby waves, by utilizing multiscale transformation and small parameter expansion, various high dimensional equations have been obtained, such as the Zakharov- Kuznetsov (ZK) equation, ${ }^{[18]}$ ZK-Burgers equation, ${ }^{[19]}$ ZKBO equation, ${ }^{[20]}$ fZK equation and Boussinesq equation, ${ }^{[21-23]} \mathrm{mKdV}$-Burgers equation and forced ZK-Burgers equation. [24,25]

In order to further explain the essence of wave motion by the obtained model equations, the analytical solutions of equations play an important role, especially the soliton solutions. Therefore, the analytical solutions of equations have always been an important research topic. For many years, people have done a lot of research in seeking the exact solution, approximate solution and numerical solution of the NPDEs. In particular, for the exact solutions of high dimensional NPDEs, researchers have obtained many methods. Such as generalized Homogeneous Balance Method, ${ }^{[26]}$, Hirota's bilinear method, ${ }^{[27-32]}$ the 
tanh-coth method, ${ }^{[33]}$ Bäcklund transformation, ${ }^{[34,35]}$ trial function method, ${ }^{[36]}$ auxiliary equation method, ${ }^{[37]}$ the Fourier Galerkin and the KarhunenCLoève (KCL) Galerkin numerical methods. ${ }^{[38]}$ However, different NPDEs have different methods. It is very important to choose a suitable method for solving the new model equation.

The main purpose of this paper is to obtain a new three dimensional equation to describe nonlinear Rossby waves. To our best knowledge, this equation is obtained for the first time. In section 2 , a new three dimensional dissipative Boussinesq equation is derived by utilizing the reduced perturbation method. Based on the new equation, the important factors of the generation of nonlinear Rossby waves are analyzed. In section 3, we explore the soliton solutions of equation and the formation mechanism of Rossby waves. In Section 4, Conclusions are presented.

\section{Derivation of the three dimensional dissipative Boussinesq equa- tion}

Starting from the shallow water wave model, it is easy to get the dimensionless QGPVE describing Rossby waves in fluid mechanics system: ${ }^{[39]}$

$$
\left(\frac{\partial}{\partial T}+\frac{\partial \Psi}{\partial X} \frac{\partial}{\partial Y}-\frac{\partial \Psi}{\partial Y} \frac{\partial}{\partial X}\right)\left(\nabla^{2} \Psi+f_{0}+\beta Y\right)=0
$$

where $\Psi$ is the total stream function, the Laplace operator $\nabla^{2}=\frac{\partial^{2}}{\partial X^{2}}+\frac{\partial^{2}}{\partial Y^{2}}, f_{0}$ and $\beta$ are constants, which are called Coriolis parameter and Rossby parameter respectively.

The dimensionless boundary condition:

$$
\frac{\partial \Psi}{\partial X}=0, Y=0,1 \text {. }
$$

Assume that the total stream function is

$$
\Psi(X, Y, T)=-\int_{0}^{Y}\left[U(s)-c_{0}+\delta \gamma\right] \mathrm{d} s+\Psi^{\prime}(X, Y, T),
$$

where $\Psi^{\prime}$ is disturbed stream function, $U(Y)$ is the zonal shear flow. where $c_{0}$ is the speed of wave. $\delta(\delta \ll 1)$ is a parameter, $\gamma$ is a detuning parameter with $O(\gamma)=1 .{ }^{[40]}$ 
We adopt the reduced perturbation method, assuming that

$$
x=\delta^{\frac{1}{2}} X, y=\delta^{\frac{1}{2}} Y, t=\delta T,
$$

and obtain

$$
\frac{\partial}{\partial X}=\delta^{\frac{1}{2}} \frac{\partial}{\partial x}, \quad \frac{\partial}{\partial Y}=\frac{\partial}{\partial Y}+\delta^{\frac{1}{2}} \frac{\partial}{\partial y}, \quad \frac{\partial}{\partial T}=\delta \frac{\partial}{\partial t},
$$

Substituting Eqs.(3)-(5) into Eqs.(1) and (2) yields

$$
\begin{gathered}
{\left[\delta^{\frac{1}{2}} \frac{\partial}{\partial t}+\left(U-c_{0}+\delta \gamma\right) \frac{\partial}{\partial x}\right]\left(\delta \frac{\partial^{2} \Psi^{\prime}}{\partial x^{2}}+\frac{\partial^{2} \Psi^{\prime}}{\partial Y^{2}}+2 \delta^{\frac{1}{2}} \frac{\partial^{2} \Psi^{\prime}}{\partial Y \partial y}+\delta \frac{\partial^{2} \Psi^{\prime}}{\partial y^{2}}\right)} \\
+\left(\beta-U^{\prime \prime}\right) \frac{\partial \Psi^{\prime}}{\partial x}+\left[\frac{\partial \Psi^{\prime}}{\partial x}\left(\frac{\partial}{\partial y}+\delta^{\frac{1}{2}} \frac{\partial}{\partial y}\right)-\left(\frac{\partial \Psi^{\prime}}{\partial Y}+\delta^{\frac{1}{2}} \frac{\partial \Psi^{\prime}}{\partial y}\right) \frac{\partial}{\partial x}\right] \\
\left(\delta \frac{\partial^{2} \Psi^{\prime}}{\partial x^{2}}+\frac{\partial^{2} \Psi^{\prime}}{\partial Y^{2}}+2 \delta^{\frac{1}{2}} \frac{\partial^{2} \Psi^{\prime}}{\partial Y \partial y}+\delta \frac{\partial^{2} \Psi^{\prime}}{\partial y^{2}}\right)=0 . \\
\frac{\partial \Psi^{\prime}}{\partial x}=0, Y=0,1 .
\end{gathered}
$$

Using the perturbation expansion method, let

$$
\Psi^{\prime}(x, Y, y, t)=\delta \Psi_{1}+\delta^{\frac{3}{2}} \Psi_{2}+\delta^{2} \Psi_{3}+\cdots .
$$

Then, we substitute Eq.(8) into Eqs.(6)and(7), and set the power of $\delta$ be zero

$$
\begin{gathered}
O\left(\delta^{1}\right):\left\{\begin{array}{c}
\left(U-c_{0}\right) \frac{\partial}{\partial x}\left(\frac{\partial^{2} \Psi_{1}}{\partial Y^{2}}\right)+\left(\beta-U^{\prime \prime}\right) \frac{\partial \Psi_{1}}{\partial x}=0, \\
\frac{\partial \Psi_{1}}{\partial x}=0, Y=0,1,
\end{array}\right. \\
O\left(\delta^{\frac{3}{2}}\right):\left\{\begin{array}{c}
\left(U-c_{0}\right) \frac{\partial}{\partial x}\left(\frac{\partial^{2} \Psi_{2}}{\partial Y^{2}}\right)+\left(\beta-U^{\prime \prime}\right) \frac{\partial \Psi_{2}}{\partial x} \\
=-\frac{\partial}{\partial t}\left(\frac{\partial^{2} \Psi_{1}}{\partial Y^{2}}\right)-2\left(U-c_{0}\right) \frac{\partial}{\partial x}\left(\frac{\partial^{2} \Psi_{1}}{\partial Y \partial y}\right), \\
\frac{\partial \Psi_{2}}{\partial x}=0, Y=0,1,
\end{array}\right. \\
O\left(\delta^{2}\right):\left\{\begin{array}{c}
\left(U-c_{0}\right) \frac{\partial}{\partial x}\left(\frac{\partial^{2} \Psi_{3}}{\partial Y^{2}}\right)+\left(\beta-U^{\prime \prime}\right) \frac{\partial \Psi_{3}}{\partial x}=-G, \\
\frac{\partial \Psi_{3}}{\partial x}=0, Y=0,1,
\end{array}\right.
\end{gathered}
$$

where

$$
\begin{gathered}
G=\left(U-c_{0}\right) \frac{\partial}{\partial x}\left(\frac{\partial^{2} \Psi_{1}}{\partial x^{2}}\right)+\frac{\partial}{\partial t}\left(\frac{\partial^{2} \Psi_{2}}{\partial Y^{2}}\right)+\gamma \frac{\partial}{\partial x}\left(\frac{\partial^{2} \Psi_{1}}{\partial Y^{2}}\right)+2 \frac{\partial}{\partial t}\left(\frac{\partial^{2} \Psi_{1}}{\partial Y \partial y}\right) \\
+2\left(U-c_{0}\right) \frac{\partial}{\partial x}\left(\frac{\partial^{2} \Psi_{2}}{\partial Y \partial y}\right)+\left(U-c_{0}\right) \frac{\partial}{\partial x}\left(\frac{\partial^{2} \Psi_{1}}{\partial y^{2}}\right) \\
+\left[\frac{\partial \Psi_{1}}{\partial x} \frac{\partial}{\partial Y}\left(\frac{\partial^{2} \Psi_{1}}{\partial Y^{2}}\right)-\frac{\partial \Psi_{1}}{\partial Y} \frac{\partial}{\partial x}\left(\frac{\partial^{2} \Psi_{1}}{\partial Y^{2}}\right)\right]
\end{gathered}
$$


We suppose that $\Psi_{1}=u(x, y, t) \Phi_{1}(Y)$, and substitute it into Eq.(9) the zonal shear flow

$$
\left\{\begin{array}{l}
\Phi_{1}^{\prime \prime}+\frac{\beta-U^{\prime \prime}}{U-c_{0}} \Phi_{1}=0, \\
\Phi_{1}(0)=\Phi_{1}(1)=0 .
\end{array}\right.
$$

where $U-c_{0} \neq 0$. For Eq.(10), assume that is

$$
\Psi_{2}=v_{1}(x, y, t) \Phi_{21}(Y)+v_{2}(x, y, t) \Phi_{22}(Y) .
$$

and substitute it into Eq.(10), combining Eq.(13) yields

$$
\begin{gathered}
\left(U-c_{0}\right)\left(v_{1 x} \Phi_{21}^{\prime \prime}+v_{2 x} \Phi_{22}^{\prime \prime}\right)+\left(\beta-U^{\prime \prime}\right)\left(v_{1 x} \Phi_{21}+v_{2 x} \Phi_{22}\right) \\
=-u_{t} \Phi_{1}^{\prime \prime}-2\left(U-c_{0}\right) u_{x y} \Phi_{1}^{\prime}
\end{gathered}
$$

where $v_{1 x}=\frac{\partial v_{1}}{\partial x}$, and other representations are similar.

To simplify the problem, we assume

$$
v_{1 x}=u_{t}, \quad v_{2}=u_{y} .
$$

Consequently, Eq. (15) transforms into the following two equation sets

$$
\begin{gathered}
\left\{\begin{array}{c}
\Phi_{21}^{\prime \prime}+\frac{\beta-U^{\prime \prime}}{U-c_{0}} \Phi_{21}=\frac{\beta-U^{\prime \prime}}{\left(U-c_{0}\right)^{2}} \Phi_{1}, \\
\Phi_{21}(0)=\Phi_{21}(1)=0 .
\end{array}\right. \\
\left\{\begin{array}{c}
\Phi_{22}^{\prime \prime}+\frac{\beta-U^{\prime \prime}}{U-c_{0}} \Phi_{22}=-2 \Phi_{1}^{\prime}, \\
\Phi_{22}(0)=\Phi_{22}(1)=0 .
\end{array}\right.
\end{gathered}
$$

For Eq.(11), using the singular elimination condition:

$$
\int_{0}^{1} \frac{\Phi_{1}}{U-c_{0}} G \mathrm{~d} Y=0 .
$$

Substituting Eq.(12) into Eq.(19), and using the boundary condition of Eqs.(13), (17) and (18), we obtain

$$
u_{t t}+e_{1} u_{x x}+e_{2}\left(u^{2}\right)_{x x}+e_{3} u_{t x y}+e_{4} u_{x x x x}+e_{5} u_{x x y y}=0 .
$$


Where

$$
\left\{\begin{array}{l}
e_{1}=-\frac{\gamma}{I} \int_{0}^{1} \frac{\beta-U^{\prime \prime}}{\left(U-c_{0}\right)^{2}} \Phi_{1}^{2} \mathrm{~d} Y \\
e_{2}=-\frac{1}{2 I} \int_{0}^{1} \frac{\Phi_{1}^{3}}{U-c_{0}} \frac{\mathrm{d}}{\mathrm{d} Y}\left(\frac{\beta-U^{\prime \prime}}{U-c_{0}}\right) \mathrm{d} Y \\
e_{3}=\frac{1}{I} \int_{0}^{1}\left(2 \Phi_{1} \Phi_{21}^{\prime}-\frac{\beta-U^{\prime \prime}}{\left(U-c_{0}\right)^{2}} \Phi_{1} \Phi_{22}\right) \mathrm{d} Y \\
e_{4}=\frac{1}{I} \int_{0}^{1} \Phi_{1}^{2} \mathrm{~d} Y \\
e_{5}=\frac{1}{I} \int_{0}^{1}\left(2 \Phi_{1} \Phi_{22}^{\prime}+\Phi_{1}^{2}\right) \mathrm{d} Y \\
I=\int_{0}^{1} \frac{\beta-U^{\prime \prime}}{\left(U-c_{0}\right)^{2}}\left(\frac{\Phi_{1}^{2}}{U-c_{0}}-\Phi_{1} \Phi_{21}\right) \mathrm{d} Y .
\end{array}\right.
$$

Remark 1: $\Phi_{1}, \Phi_{21}, \Phi_{22}$ are determined by Eqs.(13), (17) and (18). Note that Eq.(20) is a new three dimensional dissipative Boussinesq equation for describing the evolution of Rossby waves in fluid mechanics system. The new equation is different from the existing high dimensional Boussinesq equation. ${ }^{23,26,33}$ When $e_{3}=0$ and $e_{5}=0$, Eq. (20) is a two dimensional Boussinesq equation. $e_{2}$ is the nonlinear coefficient, which indicates that the bate effect and the zonal shear flow can induce solitary waves. $e_{1}, e_{3}, e_{4}$ and $e_{5}$ represents the dispersion relation of linear wave, and are related to the bate effect and the zonal shear flow .

Remark 2: Through the new equation (20), we consider the dispersion relation of linear Rossby wave by using the normal mode method. Assuming that $u(x, y, t)=u_{0} \exp (i \xi), \xi=k x+l y-\omega t$, and substituting it into the linear part of Eq.(20), yields

$$
\omega=\frac{1}{2}\left[i e_{3} k l \pm k \sqrt{\left(e_{4} k^{2}+e_{5} l^{2}\right)-\left(e_{3}^{2} k^{2}+4 e_{1}\right)}\right] .
$$

when $e_{3}<0, \Delta=\left(e_{4} k^{2}+e_{5} l^{2}\right)-\left(e_{3}^{2} k^{2}+4 e_{1}\right)>0$,

$$
u(x, y, t)=u_{0} \exp \left(\frac{1}{2} e_{3} k l t\right) \exp \left[i\left(k x+l y \pm \frac{1}{2} k \sqrt{\Delta} t\right] .\right.
$$

From Eq.(22), we can see that the dispersion relation of the linear Rossby wave described by the new three dimensional equation is relatively complex, which is close to the wave phenomenon in the real atmospheric motion. From Eq.(23), Note that $e_{3}$ has a dissipative effect, and can cause the decay of the linear Rossby wave amplitude, which further shows that beta effect and zonal shear flow affect the formation of Rossby wave. The linear Rossby wave described 
by the new equation is a dissipative wave, which is different from the previous research results.

\section{Methods and solutions}

In this section, we obtain the two kinds of soliton solutions of equation (20) by using the trial function and auxiliary equation method. For Eq.(20), Assuming that

$$
u(x, y, t)=u(\xi), \xi=k x+l y-\omega t .
$$

where $k$ and $l$ are the wave number, and $\omega$ is the wave frequency. Substituting (24) into (20), then integrating it two with respect to $\xi$, yields

$$
\left(\omega^{2}+e_{1} k^{2}\right) u+e_{2} k^{2} u^{2}-e_{3} k l \omega \frac{\mathrm{d} u}{\mathrm{~d} \xi}+\left(e_{4} k^{4}+e_{5} k^{2} l^{2}\right) \frac{\mathrm{d}^{2} u}{\mathrm{~d} \xi^{2}}=0 .
$$

\subsection{The trial function method}

We assume that

$$
u(\xi)=\frac{b_{0} \mathrm{e}^{b_{1} \xi}}{\left(1+\mathrm{e}^{b_{2} \xi}\right)^{2}} .
$$

where $b_{0}, b_{1}, b_{2}$ are constants to be determined. Substituting Eq. (26) into Eq. (25) yields

$$
\begin{gathered}
\left(\omega^{2}+e_{1} k^{2}\right)\left(1+2 \mathrm{e}^{b_{2} \xi}+\mathrm{e}^{2 b_{2} \xi}\right)+e_{2} k^{2} b_{0} \mathrm{e}^{b_{1} \xi}-e_{3} k l \omega \\
{\left[b_{1}-2\left(b_{1}-b_{2}\right) \mathrm{e}^{b_{2} \xi}+\left(b_{1}-2 b_{2}\right)^{2} \mathrm{e}^{2 b_{2} \xi}\right]+\left(e_{4} k^{4}+e_{5} k^{2} l^{2}\right)} \\
{\left[b_{1}^{2}-2\left(b_{2}^{2}-b_{1}^{2}-2 b_{1} b_{2}\right) \mathrm{e}^{b_{2} \xi}+\left(2 b_{2}-b_{1}\right)^{2} \mathrm{e}^{2 b_{2} \xi}\right]=0 .}
\end{gathered}
$$

We take $b_{1}=0$, and the coefficients of $\mathrm{e}^{b_{2} \xi}, \mathrm{e}^{2 b_{2} \xi}$ terms to zero, this leads to

$$
b_{0}=-\frac{\omega^{2}+e_{1} k^{2}}{e_{2} k^{2}}, b_{2}=\frac{5\left(\omega^{2}+e_{1} k^{2}\right)}{6 e_{3} k l \omega} .
$$

Substituting Eq.(28) into Eq. (26), the kind-type solution of Eq. (20) is

$$
u(x, y, t)=-\frac{\omega^{2}+e_{1} k^{2}}{4 e_{2} k^{2}}\left[1+\tanh \frac{5\left(\omega^{2}+e_{1} k^{2}\right)}{12 e_{3} k l \omega}(k x+l y-\omega t)\right]^{2} .
$$

where $\omega^{2}=\frac{25 e_{1} k^{2}\left(e_{4} k^{2}+e_{5} l^{2}\right)}{6 e_{3}^{2} l^{2}-25\left(e_{4} k^{2}+e_{5} l^{2}\right)}$. 

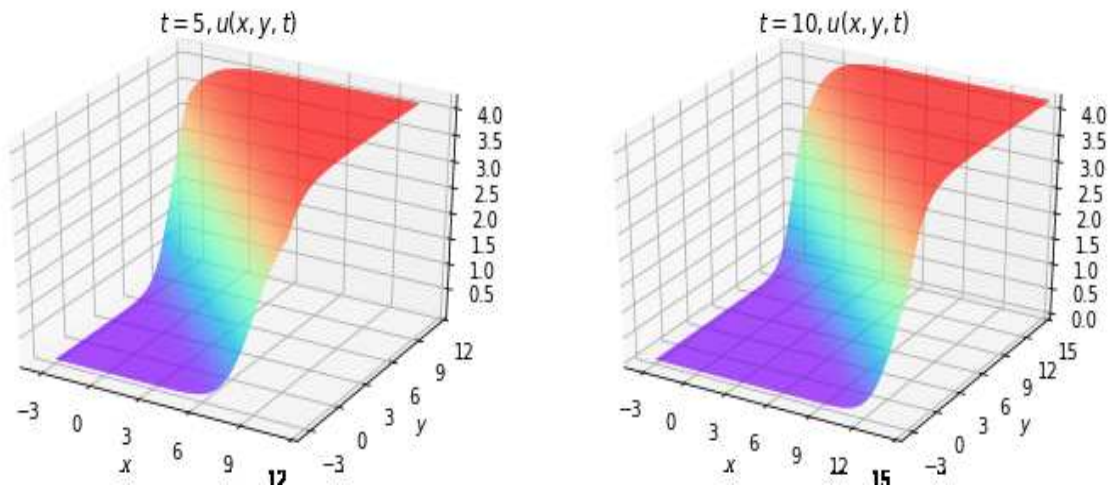

Fig.1. The evolution of solution (29), parameter as $k=l=1, e_{1}=1, e_{2}=-\frac{1}{2}$, $e_{3}=2, e_{4}=\frac{1}{4}, e_{5}=\frac{1}{4}$.

\subsection{The auxiliary equation method}

We assume that

$$
u(\xi)=\sum_{j=0}^{M} \lambda_{j} Q^{j} .
$$

where $Q=Q(\xi)$ satisfies the following equation:

$$
Q^{\prime}=a Q+b
$$

where $a, b$ are an undetermined constants. Note that Eq.(31) is called the auxiliary equation. In Ref [26], the solutions of Eq.(31) are as follows:

Case 1.when $a=-b$,

$$
Q=Q(\xi)=\tanh (b \xi)
$$

and

$$
Q=Q(\xi)=\operatorname{coth}(b \xi) .
$$

Case 2.when $a=b$

$$
Q=Q(\xi)=\tan (b \xi)
$$

and

$$
Q=Q(\xi)=-\cot (b \xi)
$$


By balancing the nonlinear term and the highest-order derivative term of (25), we take $M=2$. Substituting Eq.(30) into Eq. (25), we obtain the following algebraic equation sets

$$
\left\{\begin{array}{l}
6\left(e_{4} k^{4}+e_{5} k^{2} l^{2}\right) a^{2} \lambda_{2}+e_{2} k^{2} \lambda_{2}^{2}=0, \\
2\left(e_{4} k^{4}+e_{5} k^{2} l^{2}\right) a^{2} \lambda_{1}-2 e_{3} k l \omega a \lambda_{2}+2 e_{2} k^{2} \lambda_{1} \lambda_{2}=0, \\
8\left(e_{4} k^{4}+e_{5} k^{2} l^{2}\right) a b \lambda_{2}-e_{3} k l \omega a \lambda_{1}+e_{2} k^{2}\left(2 \lambda_{0} \lambda_{2}+\lambda_{1}^{2}\right) \\
+\left(\omega^{2}+e_{1} k^{2}\right) \lambda_{2}=0, \\
2\left(e_{4} k^{4}+e_{5} k^{2} l^{2}\right) a b \lambda_{1}-2 e_{3} k l \omega b \lambda_{2}+2 e_{2} k^{2} \lambda_{0} \lambda_{1} \\
+\left(\omega^{2}+e_{1} k^{2}\right) \lambda_{1}=0, \\
2\left(e_{4} k^{4}+e_{5} k^{2} l^{2}\right) b^{2} \lambda_{2}-e_{3} k l \omega b \lambda_{1}+e_{2} k^{2} \lambda_{0}^{2}+\left(\omega^{2}+e_{1} k^{2}\right) \lambda_{2}=0 .
\end{array}\right.
$$

For Eqs.(36), with the help of Mathematics, we obtain

$$
\left\{\begin{aligned}
\lambda_{0} & =-\frac{12 a b\left(e_{4} k^{4}+e_{5} k^{2} l^{2}\right)+\left(\omega^{2}+e_{1} k^{2}\right)}{2 e_{3} k l \omega} \\
\lambda_{1} & =\frac{6 a e_{3} k l \omega}{5 e_{2} k} \\
\lambda_{2} & =-\frac{6 a^{2}\left(e_{4} k^{2}+e_{5} l^{2}\right)}{e_{2}}
\end{aligned}\right.
$$

where $a, b$ satisfying as follows:

$$
a b=-\frac{e_{3}^{2} l^{2} \omega^{2}}{100 k^{2}\left(e_{4} k^{2}+e_{5} l^{2}\right)^{2}} .
$$

and

$$
\begin{aligned}
a^{2}= & \frac{\omega^{2}+e_{1} k^{2}}{25 k^{2}\left(e_{4} k^{2}+e_{5} l^{2}\right)}-\frac{e_{3}^{2} l^{2} \omega^{2}}{100 k^{2}\left(e_{4} k^{2}+e_{5} l^{2}\right)} \\
& +\frac{3 e_{3}^{4} l^{4} \omega^{4}}{125 k^{2}\left(e_{4} k^{2}+e_{5} l^{2}\right)\left(\omega^{2}+e_{1} k^{2}\right)} .
\end{aligned}
$$

Therefore, from Eqs.(32) and (33), we can get

$$
\begin{aligned}
u(x, y, t)= & \frac{12 b^{2}\left(e_{4} k^{4}+e_{5} k^{2} l^{2}\right)+\left(\omega^{2}+e_{1} k^{2}\right)}{2 e_{3} k l \omega} \\
& -\frac{6 b e_{3} k l \omega}{5 e_{2} k} \tanh [b(k x+l y-\omega t)] \\
& -\frac{6 b^{2}\left(e_{4} k^{2}+e_{5} l^{2}\right)}{e_{2}} \tanh ^{2}[b(k x+l y-\omega t)] .
\end{aligned}
$$


and

$$
\begin{aligned}
u(x, y, t)= & \frac{12 b^{2}\left(e_{4} k^{4}+e_{5} k^{2} l^{2}\right)+\left(\omega^{2}+e_{1} k^{2}\right)}{2 e_{3} k l \omega} \\
& -\frac{6 b e_{3} k l \omega}{5 e_{2} k} \operatorname{coth}[b(k x+l y-\omega t)] \\
& -\frac{6 b^{2}\left(e_{4} k^{2}+e_{5} l^{2}\right)}{e_{2}} \operatorname{coth}^{2}[b(k x+l y-\omega t)] .
\end{aligned}
$$

where $b^{2}=\frac{e_{3}^{2} l^{2} \omega^{2}}{100 k^{2}\left(e_{4} k^{2}+e_{5} l^{2}\right)^{2}}, \omega^{2}=\frac{25 e_{1} k^{2}\left(e_{4} k^{2}+e_{5} l^{2}\right)}{6 e_{3} l^{2}-25\left(e_{4} k^{2}+e_{5} l^{2}\right)}$.

From Eqs.(34) and(35), we can also get

$$
\begin{aligned}
u(x, y, t)= & \frac{12 b_{*}^{2}\left(e_{4} k^{4}+e_{5} k^{2} l^{2}\right)+\left(\omega^{2}+e_{1} k^{2}\right)}{2 e_{3} k l \omega} \\
& \pm \mathrm{i} \frac{6 b_{*} e_{3} k l \omega}{5 e_{2} k} \tanh \left[b_{*}(k x+l y-\omega t)\right] \\
& -\frac{6 b_{*}^{2}\left(e_{4} k^{2}+e_{5} l^{2}\right)}{e_{2}} \tanh ^{2}\left[b_{*}(k x+l y-\omega t)\right] .
\end{aligned}
$$

and

$$
\begin{aligned}
u(x, y, t)= & -\frac{12 b_{*}^{2}\left(e_{4} k^{4}+e_{5} k^{2} l^{2}\right)+\left(\omega^{2}+e_{1} k^{2}\right)}{2 e_{3} k l \omega} \\
& \pm \mathrm{i} \frac{6 b_{*} e_{3} k l \omega}{5 e_{2} k} \operatorname{coth}\left[b_{*}(k x+l y-\omega t)\right] \\
& +\frac{6 b_{*}^{2}\left(e_{4} k^{2}+e_{5} l^{2}\right)}{e_{2}} \operatorname{coth}^{2}\left[b_{*}(k x+l y-\omega t)\right] .
\end{aligned}
$$

where $b^{2}=-b_{*}^{2}, b_{*}^{2}=\frac{e_{3}^{2} l^{2} \omega^{2}}{100 k^{2}\left(e_{4} k^{2}+e_{5} l^{2}\right)^{2}}, \omega^{2}=\frac{25 e_{1} k^{2}\left(e_{4} k^{2}+e_{5} l^{2}\right) i}{6 e_{3} l^{2} \pm 25\left(e_{4} k^{2}+e_{5} l^{2}\right) i}$. i is an imaginary unit.
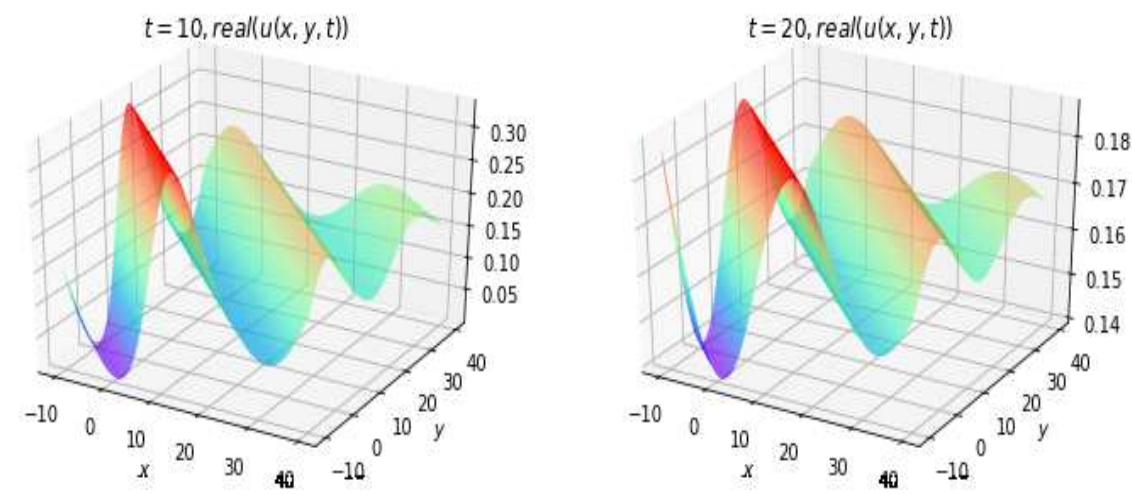

Fig.2. The evolution of real part of solution (42), parameter as $k=l=1$, $e_{1}=1, e_{2}=-\frac{1}{2}, e_{3}=\frac{3}{2}, e_{4}=\frac{1}{2}, e_{5}=1$. 
Remark 3: From Eqs.(29),(40)-(43), we can find that there are the analytical solutions of equation (20) when $e_{2}$ and $e_{3}$ are not equal to zero. Combined with the analysis of Remark 1 and 2, it is shown that beta effect and zonal shear flow have important influence on the propagation of three dimensional Rossby waves.
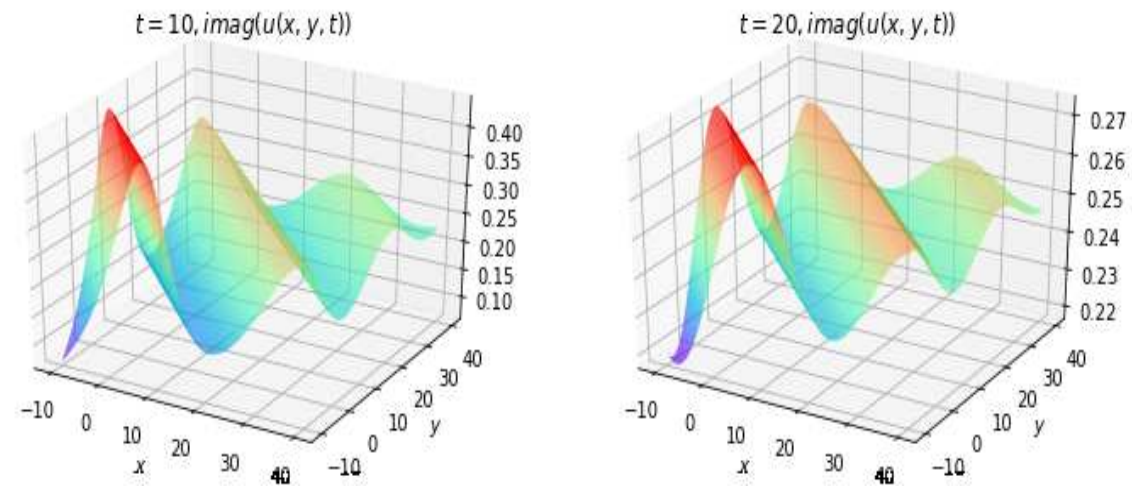

Fig.3. The evolution of imaginary part of solution (42), parameter as $k=l=1$, $e_{1}=1, e_{2}=-\frac{1}{2}, e_{3}=\frac{3}{2}, e_{4}=\frac{1}{2}, e_{5}=1$.
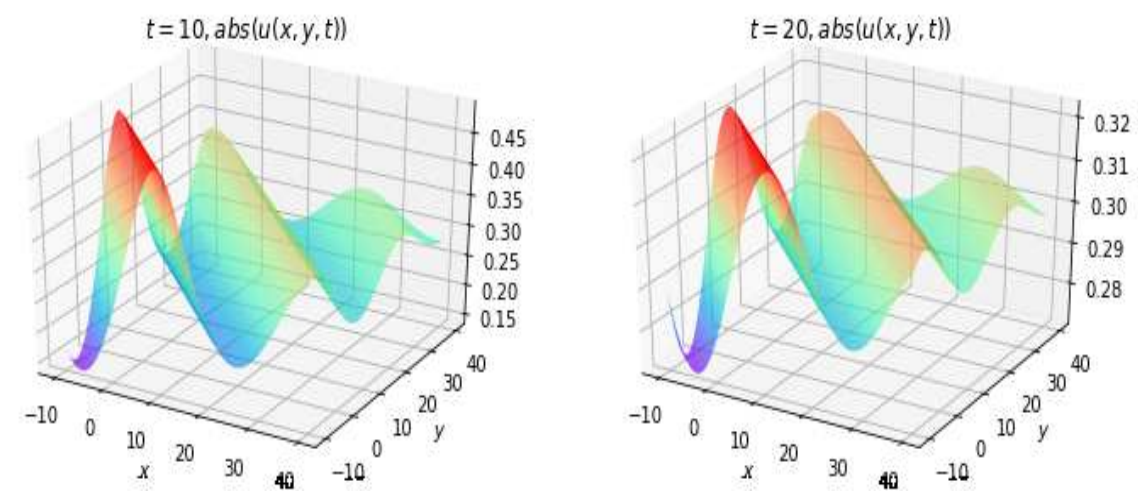

Fig.4. The evolution of module of solution (42), parameter as $k=l=1$, $e_{1}=1, e_{2}=-\frac{1}{2}, e_{3}=\frac{3}{2}, e_{4}=\frac{1}{2}, e_{5}=1$.

From Eqs.(29) and Fig.1, we can see that the solution of equation (20) is the kind-type soliton solution. From Fig.2-4, we can also see that the multiple soliton solutions of equation (20) are represented by equation (42). 


\section{Conclusion}

We obtain a new three dimensional dissipative Boussinesq equation for describing nonlinear Rossby waves. For the new three dimensional equation for Rossby waves, we explore the dispersion relation of linear Rossby wave by the normal mode method, and get the conclusion that the three dimensional linear Rossby waves is a dissipative wave. Further, we obtained the kind-type soliton and multiple soliton solutions of new equation with the aid of the trial function method and auxiliary equation method, respectively. From the result of the solution and the theoretical analysis, we draw conclude that the beta effect and zonal shear flow have important effects on the formation and decay of three

dimensional nonlinear Rossby waves in complex atmospheric motion, they are two important factors in the formation mechanism of Rossby waves.

\section{Acknowledgements}

This work was supported by the National Natural Science Foundation of China (Grant Nos.12062017, 11762011) and Research Program of Science at Universities of Inner Mongolia Autonomous Region, China(Grant NO. NJZY21272).

\section{Conflict of interest}

The authors have no conflict of interest concerning the publication of this manuscript.

\section{References}

[1] Lim,H.,Chang,C.P.: Dynamics of teleconnections and Walker circulations forced by equatorial heating. J. Atmos. Sci. 40,1897-1915(1983)

[2] Luo,D.H.,Ji,L.R.: A theory of blocking formation in the atmosphere. Sci. China 33,323-333(1989) 
[3] Wang,B.,Rui,H.: Dynamics of the coupled moist Kelvin-Rossby wave on the equatorial-plane. J. Atmos. Sci.47,397-413(1990)

[4] Horel,J.D.,Wallace,J.M.: Planetary-scale atmospheric phenomena associated with the Southern Oscillation. Mon. Weather. Rev. 109,813-829(1981)

[5] Lau,K.M.,Shen,S.: On the dynamics of intraseasonal oscillations and ENSO. J. Atmos. Sci.45,1781-1797(1988)

[6] Long,R.R.: Solitary waves in the westerlies. J. Atmos. Sci.21,197-200(1964)

[7] Wadati,M.: The modified Korteweg-de Vries equation. J. Phys. soc. Jpn.34,1289-1296(1973)

[8] Redekopp,L.G.: On the theory of solitary Rossby waves. J. Fluid. Mech. 82,725-745(1977)

[9] Redekopp,L.G.: Weidman PD. Solitary Rossby waves in zonal shear flows and their interactions. J. Atmos. Sci 35,790-804(1978)

[10] Luo,D.H.: On the Benjamin-Ono equation and its generalization in the atmosphere. Sci. China. B 32,1233(1989)

[11] Ono,H.: Algebraic Rossby wave soliton. J. Phys. Soc. Jpn.50,27572761(1981)

[12] Meng,L.,Lv, K.L.: Dissipation and algebraic solitary long-wave excited by localized topography. Chin. J. Comput. Phys. 19,259-267(2002)

[13] Yin,X.J.,Yang,L.G.,Liu,Q.S.,Su,J.M.,Wu,G.R.: Structure of equatorial envelope Rossby solitary waves with complete Coriolis force and the external source. Chaos. Solitons. Fractals. 111,68-74(2018)

[14] Meng,L.,Lv,K.L.: Nonlinear long-wave disturbances excited by localized forcing. Chin. J.Comput. Phys. 17,159-167(2000)

[15] Lu,C.N.,Fu,C.,Yang,H.W.: Time-fractional generalized Boussinesq equation for Rossby solitary waves with dissipation effect in stratified fluid and 
conservation laws as well as exact solutions. Appl.Math.Comp. 327,104116(2018)

[16] Yang,H.W.,Yin,B.S.,Shi,Y.L.: Forced dissipative Boussinesq equation for solitary waves by unstable topography. Nonlinear Dyn.70,1389-1396(2012)

[17] Yang,H.W.,Fu,C.,Sun,J.C.: Time-fractional Benjamin-Ono equation for algebraic gravity solitary waves in baroclinic atmosphere and exact multisoliton solution as well as interaction. Commun.Non.Sci.Numer.Simul.71,187201(2019)

[18] Gottwalld,G.A.: The Zakharov-Kuznetsov equation as a two-dimensional model for nonlinearRossby waves. arxiv : org /abs/nlin/031(2009)

[19] Yang,H.W.,Xu,Z.H.,Yang,D.Z.,Feng,X.R.,Yin,B.S.,Dong,H.H.: $\quad$ ZKBurgers equation for three-dimensional Rossby solitary waves and its solutions as well as chirp effect. Adv.Differ. Equ. 2016,167(2016)

[20] Yang,H.W.,Chen,X.,Guo,M.et al.: A new ZK-BO equation for threedimensional algebraic Rossby solitary waves and its solution as well as fission property. Nonlinear Dyn 91, 2019-2032 (2018)

[21] Zhang,R.G.,Yang,L.G.,Song,J.,Liu,Q.S.:(2+1)-dimensional nonlinear Rossby solitary waves under the effects of generalized beta and slowly varying topography.Nonlinear.Dyn.90,815-822(2017)

[22] Zhang,R.G.,Yang,L.G.,Song,J.,Yang,H.L.:(2+1)-dimensional Rossby waves with complete Coriolis force and its solution by homotopy perturbation method. Comput.Math.App.73,1996-2003(2017)

[23] Zhang,R.G.,Liu,Q.S.,Yang,L.G.: New model and dynamics of higherdimensional nonlinear Rossby waves. Modern.Physics. Letters B. 1950342(2019)

[24] Chen,L.G.,Yang,L.G.,Zhang,R.G.,Cui,J.F.: Generalized(2+1)-dimensional mKdV-Burgers equation and its solution by modified hyperbolic function expansion method. Results. in. Physics. 13,102280(2019) 
[25] Chen,L.G.,Yang,L.G.,Zhang,R.G.,Liu,Q.S.,Cui,J.F.: A (2+1)-dimensional nonlinear model for Rossby waves in stratified fluids and its solitary solution. Commun. Theor. Phys. 72,045004(2020)

[26] Chen,Y.,Yan,Z.Y.,Zhang,H.G.: New explicit solitary wave solutions for $(2+1)$-dimensional Boussinesq equation and (2+1)-dimensional KP equation. Phys Lett A. 307,107-113(2003)

[27] Cao,Y.,He,J.,Mihalache,D.: Families of exact solutions of a new extended (2+1)-dimensional Boussinesq equation. Nonlinear Dyn. 91, 2593-2605(2018)

[28] Wazwaz,A.M.,Kaur,L.: New integrable Boussinesq equations of distinct dimensions with diverse variety of soliton solutions. Nonlinear. Dyn. 97,8394(2019)

[29] Yu,J.,Wang,F.,Ma,W.et al.: Multiple-soliton solutions and lumps of a (3+1)-dimensional generalized KP equation. Nonlinear Dyn. 95, 16871692(2019)

[30] Lan,Z.Z.,Guo,B.L.: Nonlinear waves behaviors for a coupled generalized nonlinear SchrödingerCBoussinesq system in a homogeneous magnetized plasma. Nonlinear Dyn. 100, 3771-3784(2020)

[31] Gai,L.,Ma,W.X.,Li,M.: Lump-type solution and breather lumpCkink interaction phenomena to a $(3+1)$-dimensional GBK equation based on trilinear form. Nonlinear Dyn. 100, 2715-2727(2020)

[32] Zhao,Z.,He,L.: M-lump, high-order breather solutions and interaction dynamics of a generalized $(2+1)$-dimensional nonlinear wave equation. Nonlinear Dyn. 100, 2753-2765(2020)

[33] Ali,A.,Seadawy,A.R.,Lu,D.: Dispersive solitary wave soliton solutions of $(2+1)$-dimensional Boussineq dynamical equation via extended simple equation method.- Journal of King Saud University-Science. 31, 653-658 (2019) 
[34] Gao,L.Na.,Zi,Y.Y.,Yin,Y.H.,Ma,W.X.,Lv,X.: Backlund transformation, multiple wave solutions and lump solutions to a $(3+1)$-dimensional nonlinear evolution equation, Nonlinear. Dyn.89, 2233-2240(2017)

[35] Zhang,Y.,Chen,D.Y.: A modified Backlund transformation andmultisoliton solution for the Boussinesq equation. Chaos, Solitons.Fractals. 23, $175-181(2005)$

[36] Liu,S.K.,Fu,Z.T.,Liu,S.D.,Zhao,Q.: A simple fast method in finding particular solutions of some nonlinear PDE. Appl.Math.Mech.22,326-331(2001)

[37] Abdel Rady,A.S.,Osman,E.S.,Khalfallah,M.: On soliton solutions of the (2+1)-dimensional Boussinesq equation.Appl.Math.Comput.219,3414$3419(2012)$

[38] Smaoui,N.,Al Jamal,R.: Dynamics and control of the modified generalized Korteweg-de Vries-Burgers equation with periodic boundary conditions. Nonlinear Dyn.103, 987-1009(2021)

[39] Pedlosky,J.: Geophysical Fluid Dynamics. Springer, New York.604(1979)

[40] Warn,T.,Brasnett,B.: The amplification and capture of atmospheric solitons by topography: A theory of the onset of regional blocking. J.Atmos. Sci. 40,28-38(1983) 
Figures
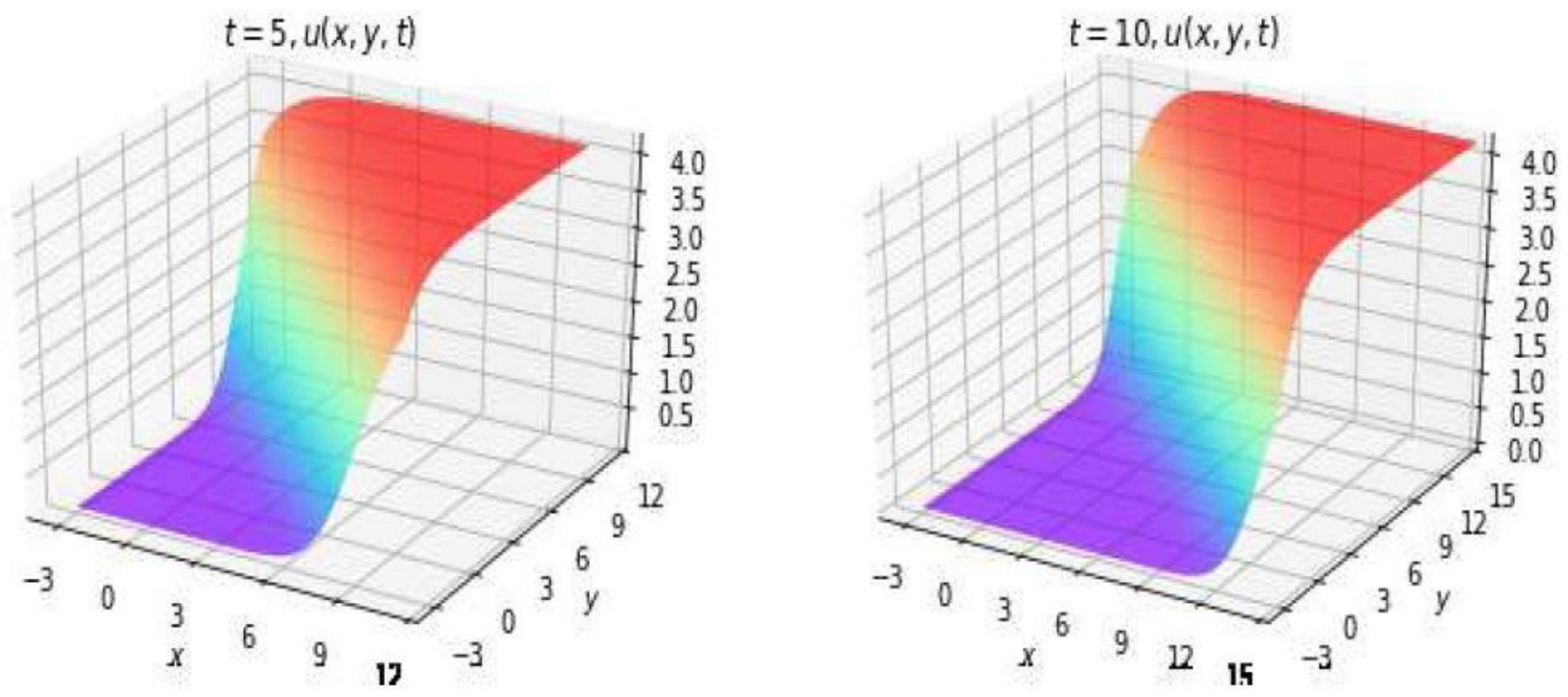

Figure 1

"Please see the Manuscript PDF file for the complete figure caption".
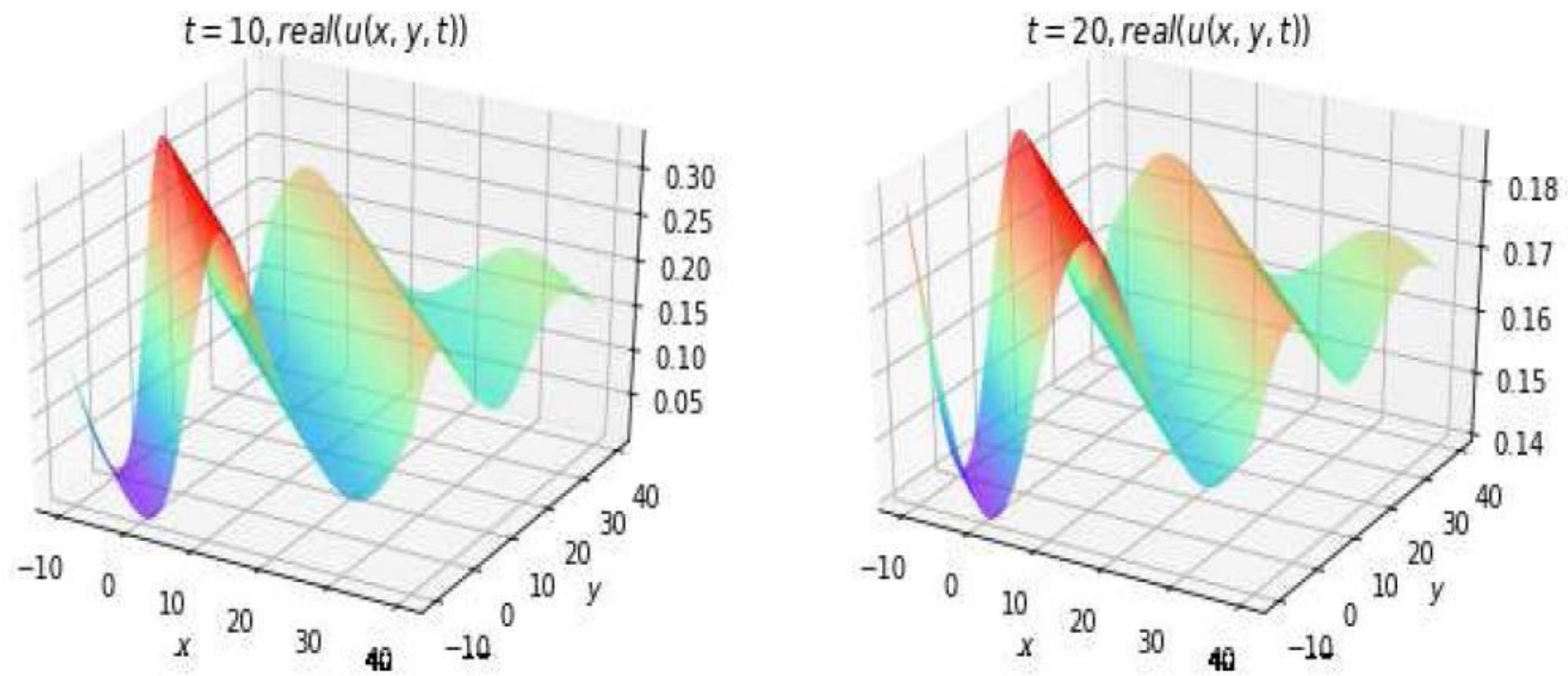

Figure 2

"Please see the Manuscript PDF file for the complete figure caption". 

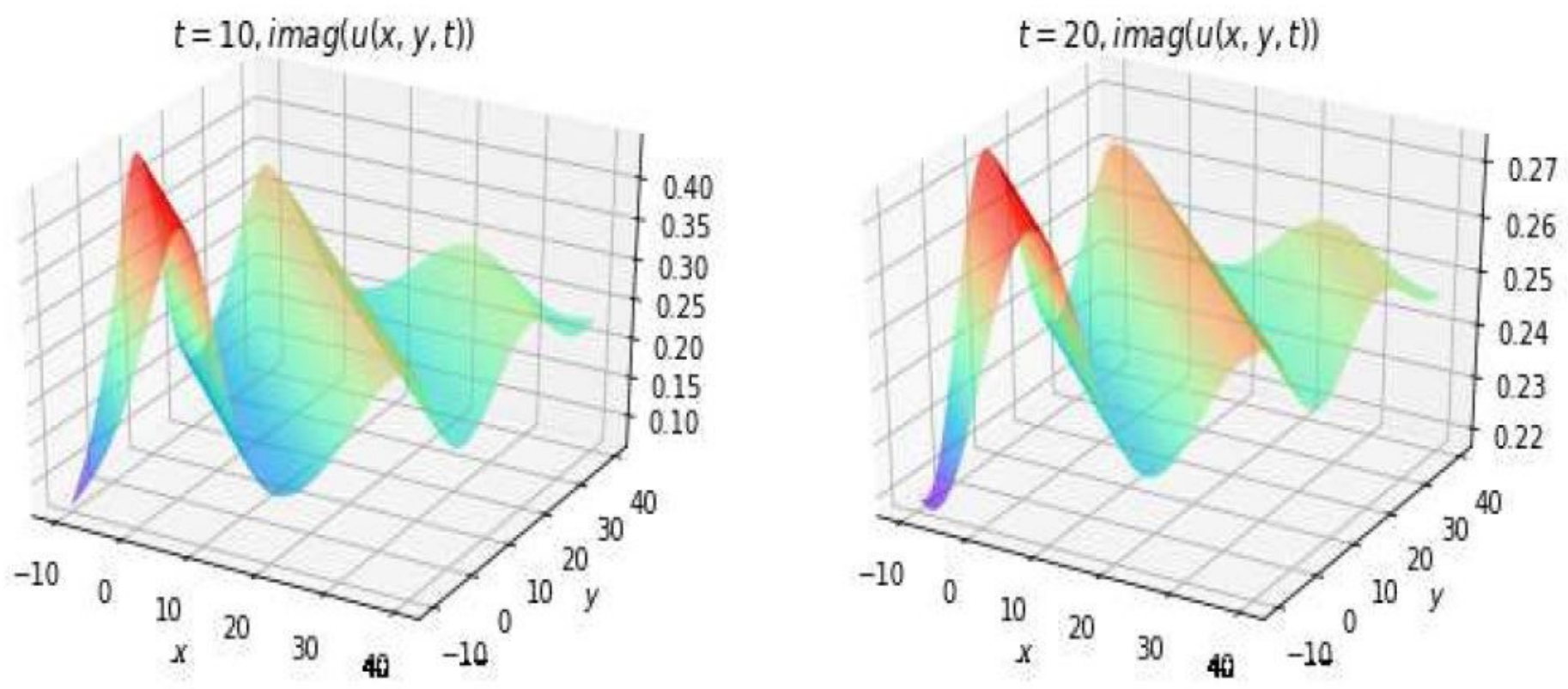

Figure 3

"Please see the Manuscript PDF file for the complete figure caption".
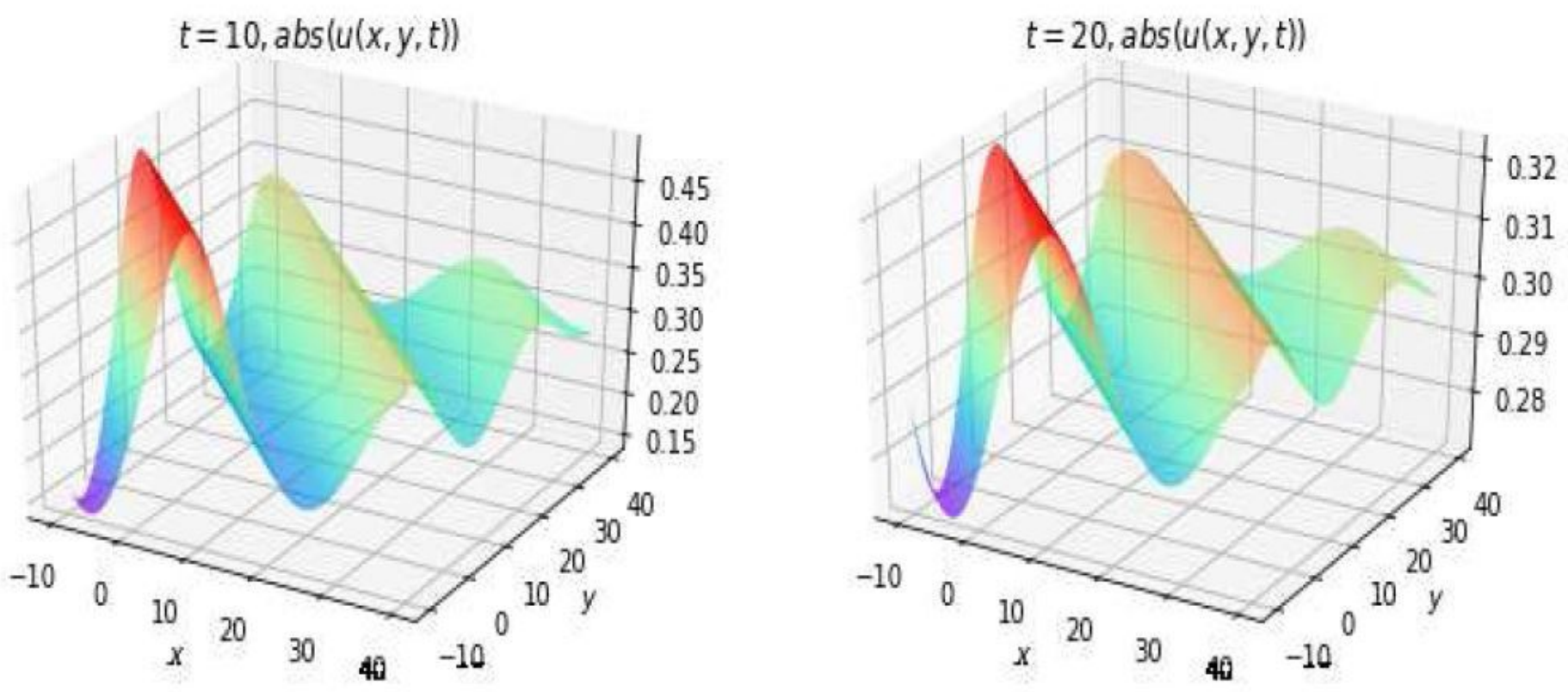

Figure 4

"Please see the Manuscript PDF file for the complete figure caption". 